ECCOMAS

Proceedia
COMPDYN 2017

$6^{\text {th }}$ ECCOMAS Thematic Conference on Computational Methods in Structural Dynamics and Earthquake Engineering

M. Papadrakakis, M. Fragiadakis (eds.)

\title{
EFFECTS OF EQUIVALENT RADIAL ACCELEROGRAMS FOR BIDIRECTIONAL SEISMIC EVENTS ON BASE-ISOLATED STRUCTURES
}

\author{
M. Furinghetti ${ }^{1}$, A. Pavese $^{2}$, and A. Foti ${ }^{1}$ \\ ${ }^{1}$ EUCENTRE - Pavia \\ via Ferrata 1, 27100 Pavia - Italy \\ e-mail: marco.furinghetti@eucentre.it \\ ${ }^{2}$ University of Pavia \\ via Ferrata 3, 27100 Pavia - Italy \\ e-mail: a.pavese@unipv.it
}

Keywords: Equivalent Uniaxial Accelerogram, Bidirectional motion, base-isolation, Concave Surface Slider, bi-axial interaction, frictional force.

\begin{abstract}
A general seismic event can be considered as the superposition of two individual time histories of ground acceleration, acting simultaneously at the base of a structural system, along the main directions of a given reference system. Response spectra can be computed either for each component, or for the global seismic event, by considering a Single Degree of Freedom with different values of self-period, subjected to both the $x$ and $y$ accelerograms. Such a bi-directional response spectrum has higher spectral coordinates with respect to the individual ones, because of the instantaneous contributions of both directions of ground motion in the structural response. Nonetheless, an earthquake is expected to release most of the total energy of the event along an actual radial direction, mainly due to the propagation direction of seismic waves from the hypocenter toward the ground surface, even though the ground motion develops according to a random trajectory. A recent research work proposed an analytical procedure for the computation of an "equivalent uniaxial accelerogram" for a bidirectional earthquake: the proposed theory allows to compute a single ground acceleration time-history, related to a proper direction angle, able to reproduce the same effects of a two-components seismic event on a base-isolated structural system: results showed a good agreement between the equivalent radial and the bidirectional structural responses, even if non-negligible differences were observed. In this work a more refined procedure is proposed for the definition of the equivalent unidirectional accelerogram for a base-isolated structure. A wide validation study is reported, by analyzing 100 bidirectional seismic events: two different ranges for vibration period have been assumed, in order to evaluate the accuracy of the overall procedure, by considering a large rather than a small portion of the reference response spectrum. Finally, a case study structure has been modeled, to underline effects of the equivalent radial response, in comparison to the reference bidirectional case.
\end{abstract}




\section{INTRODUCTION}

The non-linear time history analysis represents the most realistic structural analysis typology which can be considered: a structural F.E.M. can be subjected to real recordings of ground accelerations along the main directions of motion, and so internal actions of each structural element can be stepwise computed and checked. Generally, seismic events can be considered as three individual translational ground motions, simultaneously acting at the base of a structural system, since ground rotational degrees of freedom can be neglected [1]. In most of cases, also the vertical component is not modeled, since its effects on the main response parameters are not comparable to the ones induced by horizontal seismic motions. On the other hand, unidirectional approaches are much more common among practitioners, given the higher level of simplicity. Standard codes provide prescriptions for directional combinations of radial response results, whereas some guidelines for selection of real and spectrummatched single component accelerograms can be found in the literature [9]. When bidirectional earthquakes are considered, the response spectrum should be able to represent a two component seismic event as a single response parameter. Different combinations of the single-component response spectra can be found, such as the geometric mean, rather than the arithmetic mean or the Square Root of the Sum of Squares (SRSS), although the geometric mean seems to be the most commonly used, also for Ground Motion Prediction Equations. Also for base-isolated structures, it is important to reproduce the ground shaking conditions in the best and most realistic way possible. Concerning friction-based isolators, it is well known that the hysteretic behavior is affected by the shape of the trajectory of the devices, due to the stepwise projection of the friction force along the main directions of motion $[5,10,11,12]$. However, especially in the design phase, unidirectional approaches are assumed, and bilinearized hysteretic constitutive laws are considered to design/check the implemented isolators $[2,4]$. Moreover, it is possible to compute an equivalent damping ratio of the isolation system by considering a symmetric unidirectional hysteretic loop at the maximum displacement, which is actually an unknown at the beginning, so that an iterative procedure has to be solved. In addition, standard codes for testing isolation friction-based devices provide expressions for an average friction coefficient per cycle, under unidirectional sinusoidal motion [3], and no prescriptions for bidirectional tests are given.

In the present endeavor a refined procedure for the computation of an equivalent ground acceleration time series is presented, for bidirectional seismic events: precisely, an optimum orientation angle is computed, by minimizing the Mean Square Error between a bidirectional reference response spectrum and a radial one, related to the projection of both the planar components along the considered direction. Two different period ranges for the computation of the MSE value have been analyzed, in order to highlight the effect of a smaller rather than a larger period range. The presented procedure has been applied to a selection of 100 individual bidirectional earthquakes, and results have been statistically studied. Finally, a case study base-isolated structure has been modeled, and bidirectional Non Linear Time History Analyses have been performed: results have been compared to the effects of equivalent radial motions, in terms of peak vectorial response parameters, for both the isolation system (displacement and force demands) and the building structure (displacement, absolute acceleration and interstorey shear profiles).

Results have shown that the structural response returned by the equivalent unidirectional ground motions provides a very good estimate of the seismic behavior of the system, when subjected to bidirectional motions. 


\section{DEFINITION OF THE EQUIVALENT RADIAL EVENT}

In this section a refined procedure for the evaluation of an equivalent radial accelerogram for bidirectional earthquakes is proposed, as a subsequent development of a previous study on the seismic response of base isolated structures under general seismic motions [6]. According to the aforementioned theory, it is possible to determine an actual horizontal direction for a radial motion, which can reproduce approximately same effects of a bidirectional seismic event on a structural system, even though a preferential direction of motion can not be recognized, by considering the ground acceleration diagram (Figure 1, left).
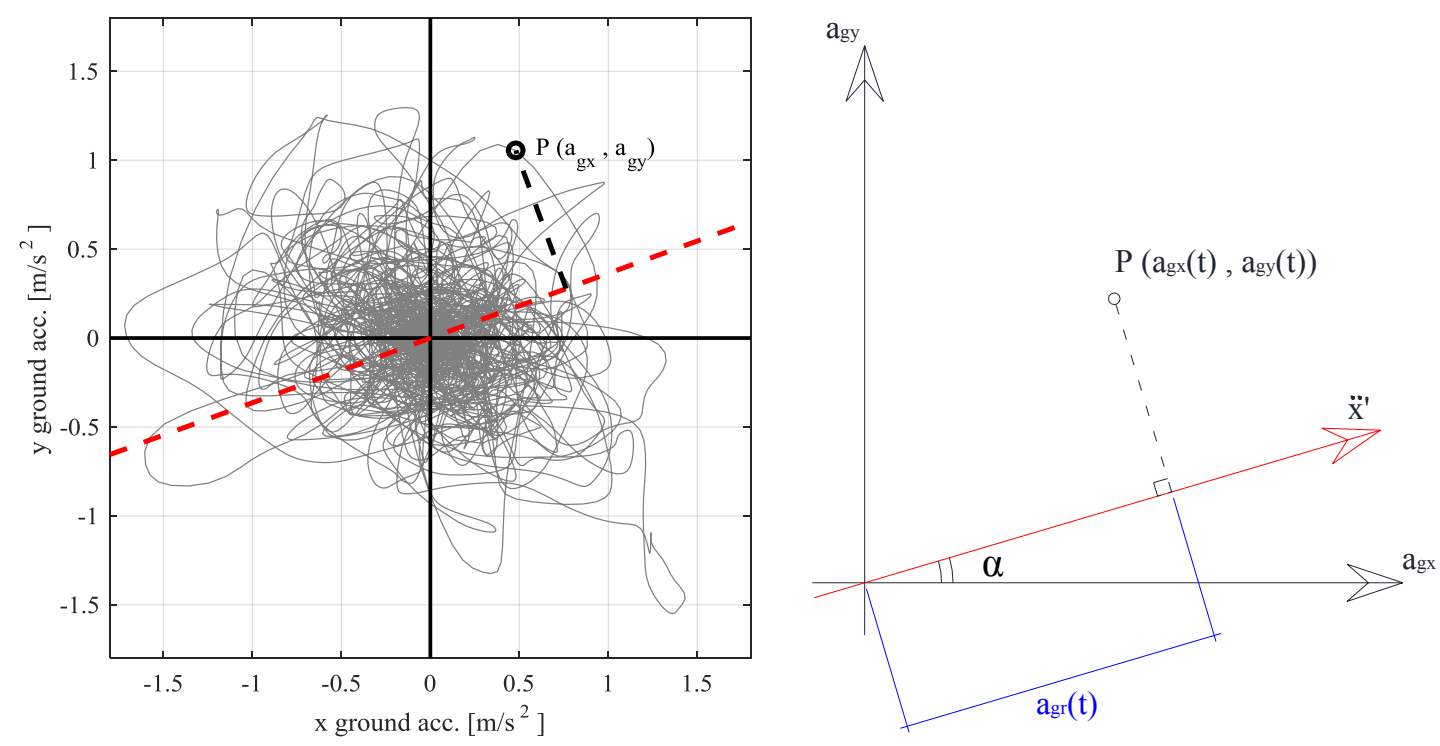

Figure 1: Acceleration points projection scheme - Irpinia earthquake 1980.

Effects of the reference bidirectional earthquake for each case is considered in terms of bidirectional response spectra: since a general planar seismic event can be represented by a couple of ground acceleration signals along two orthogonal horizontal directions, reference displacement and acceleration spectra are computed by considering for every period values a Single Degree of Freedom system, subjected to both $\mathrm{x}$ and $\mathrm{y}$ components simultaneously. Thus, for a given damping ratio $\xi$, the following equations of motion can be defined and timeintegrated:

$$
\begin{aligned}
& \ddot{x}+2 \xi \omega \dot{x}+\omega^{2} x=-\ddot{x}_{g} \\
& \ddot{y}+2 \xi \omega \dot{y}+\omega^{2} y=-\ddot{y}_{g}
\end{aligned}
$$

Hence, Spectral coordinates can be computed, by collecting maximum vectorial displacement and absolute acceleration.

$$
\begin{aligned}
& S_{d}^{2 D}(\xi, T)=\max \left(\sqrt{x^{2}+y^{2}}\right) \\
& S_{a}^{2 D}(\xi, T)=\max \left(\sqrt{\left(\ddot{x}+\ddot{x}_{g}\right)^{2}+\left(\ddot{y}+\ddot{y}_{g}\right)^{2}}\right)
\end{aligned}
$$

In Figure 1 (right) the projection procedure is shown: for a given orientation angle all the acceleration points are projected along the considered direction. Therefore, the radial projection of the bidirectional earthquake $\left.\mathrm{agr}_{\mathrm{gr}} \mathrm{t}\right)$ is defined as follows:

$$
a_{g r}(t)=a_{g x}(t) \cdot \cos (\alpha)+a_{g y}(t) \cdot \sin (\alpha)
$$


The optimum value for the orientation angle is computed, by minimizing the square root of the Mean Square Error (MSE) between the reference bidirectional and the equivalent radial spectra, both displacement and absolute acceleration, for a given period range.

$$
\begin{aligned}
& E^{S_{d}}(\xi, \alpha)=\sqrt{M S E^{S_{d}}}=\sqrt{\frac{1}{n_{T}} \sum_{i=1}^{n_{T}}\left(S_{d}^{2 D}\left(\xi, T_{i}\right)-S_{d}^{1 D}\left(\xi, \alpha, T_{i}\right)\right)^{2}} \\
& E^{S_{a}}(\xi, \alpha)=\sqrt{M S E^{S_{a}}}=\sqrt{\frac{1}{n_{T}} \sum_{i=1}^{n_{T}}\left(S_{a}^{2 D}\left(\xi, T_{i}\right)-S_{a}^{1 D}\left(\xi, \alpha, T_{i}\right)\right)^{2}}
\end{aligned}
$$

Where $\mathrm{n}_{\mathrm{T}}$ is the total number of structural periods, contained in the considered period range. A previous study has reported results related to a full range of periods, that is from $0.0 \mathrm{sec}$ to $4.0 \mathrm{sec}$ (Furinghetti and Pavese, 2017); generally, given the high variability of spectral coordinates in the acceleration plateau, better results are expected, if a smaller period range is assumed. Thus, in the present endeavor the period range has been defined, according to prescriptions of the Italian Building Code (I.B.C.) for spectrum-compatibility: period range for base-isolated structures is assumed bounded between $0.15 \mathrm{sec}$ and $1.2 \mathrm{~T}_{\text {is }}$, where $\mathrm{T}_{\text {is }}$ is the secant period of the base-isolated structure. In Figure 2 different period ranges are shown for Irpinia earthquake 1980, by assuming an isolated secant period of 2,5 sec.

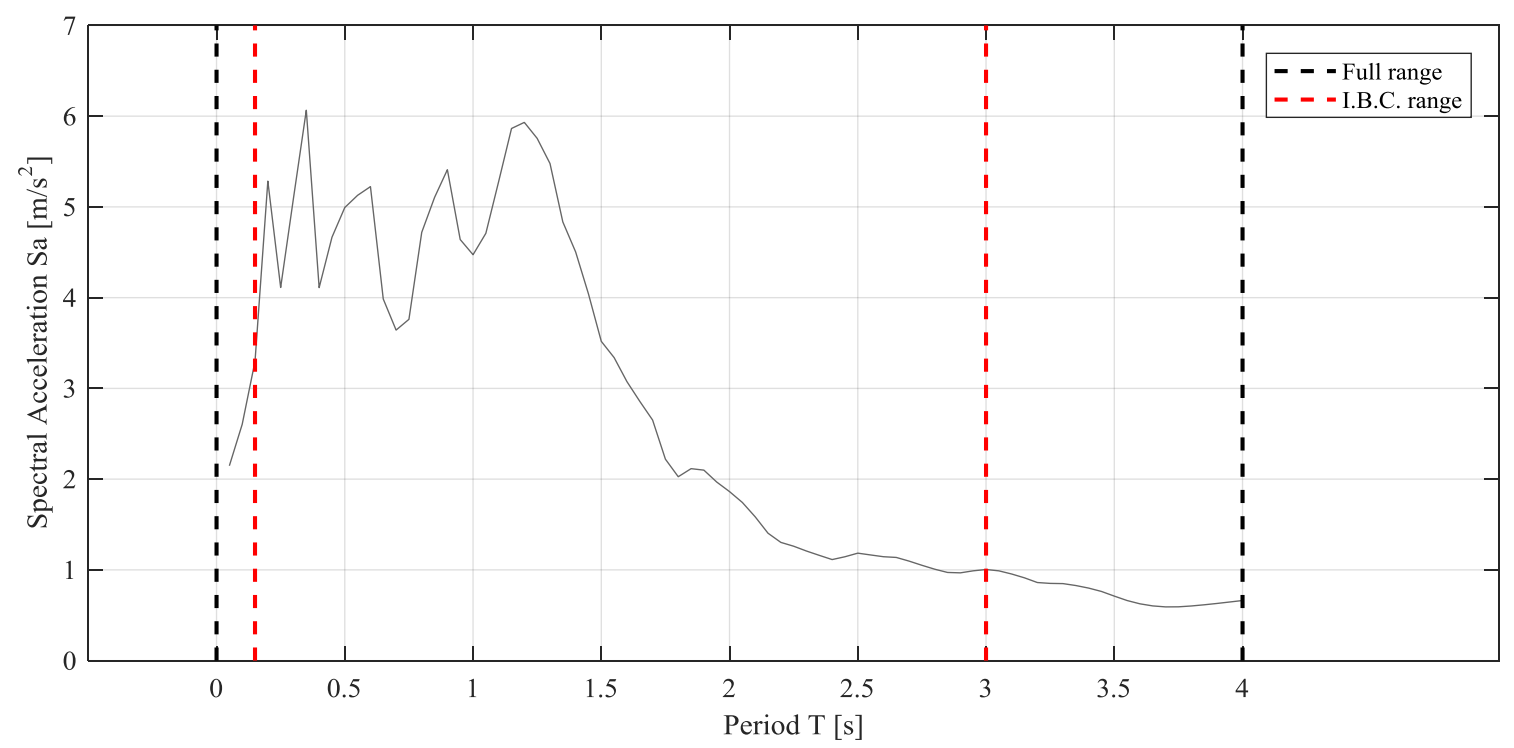

Figure 2: Period ranges for 5\% damping response spectrum - Irpinia earthquake 1980.

Thus, equivalent radial accelerograms can be also adopted, when non-linear time history analyses are performed, by checking spectrum compatibility of 7 equivalent signals with respect to the target spectrum.

\section{STATISTICAL ANALYSIS OF BIDIRECTIONAL EVENTS}

In this section the comparison of the previously defined period range definitions is reported, by considering 100 bidirectional seismic events, which have been selected from databases of the program REXEL [9]:

- ESD: European Strong-motion Database;

- ITACA: Italian Accelerometric Archive; 
- SIMBAD v3.0: Selected Input Motions for displacement-Based Assessment and Design.

A selection and scaling procedure has been applied in order to consider a high displacement demand, since base-isolated structure are considered: precisely, the vectorial displacement demand of a Single Degree Of Freedom system (SDOF) has been computed for each seismic event, by considering the actual non-linear constitutive law of a bidirectional isolation system of Curved Surface Slider (CSS) devices [7]:

$$
\begin{aligned}
& \ddot{x}+g\left\{\frac{x}{R_{e q}}+\mu \cdot \tanh \left(\frac{\sqrt{\dot{x}^{2}+\dot{y}^{2}}}{v_{s}}\right) \cdot \frac{\dot{x}}{\sqrt{\dot{x}^{2}+\dot{y}^{2}}}\right\}=-\ddot{x}_{g} \\
& \ddot{y}+g\left\{\frac{y}{R_{e q}}+\mu \cdot \tanh \left(\frac{\sqrt{\dot{x}^{2}+\dot{y}^{2}}}{v_{s}}\right) \cdot \frac{\dot{y}}{\sqrt{\dot{x}^{2}+\dot{y}^{2}}}\right\}=-\ddot{y}_{g}
\end{aligned}
$$

With such a hysteretic model, it is possible to account for the actual biaxial interaction of the directions of motion, for the stepwise projection of the main frictional force, which is assumed to be parallel to the trajectory of the isolation system. Then, records have been scaled in order to achieve a displacement demand bounded between $\pm 20 \%$ of the design displacement of the considered device $(200,0 \mathrm{~mm})$; a constant friction coefficient $\mu$ equal to $7 \%$ and an equivalent radius of curvature $R_{\mathrm{eq}}$ equal to $3,0 \mathrm{~m}$ have been assumed. A small variation range for scale factors has been considered, in order not to obtained unrealistic ground acceleration signals: all scale factors have been bounded between 0,5 and 2,0.

In Figure 3 results of the selected records are reported, in terms of peak displacements and scale factors. Thus, for the selected events, the optimum orientation angle has been computed, by considering both acceleration and displacement approaches, for several damping ratio values; furthermore, results related to full-range of period and I.B.C. range have been compared.
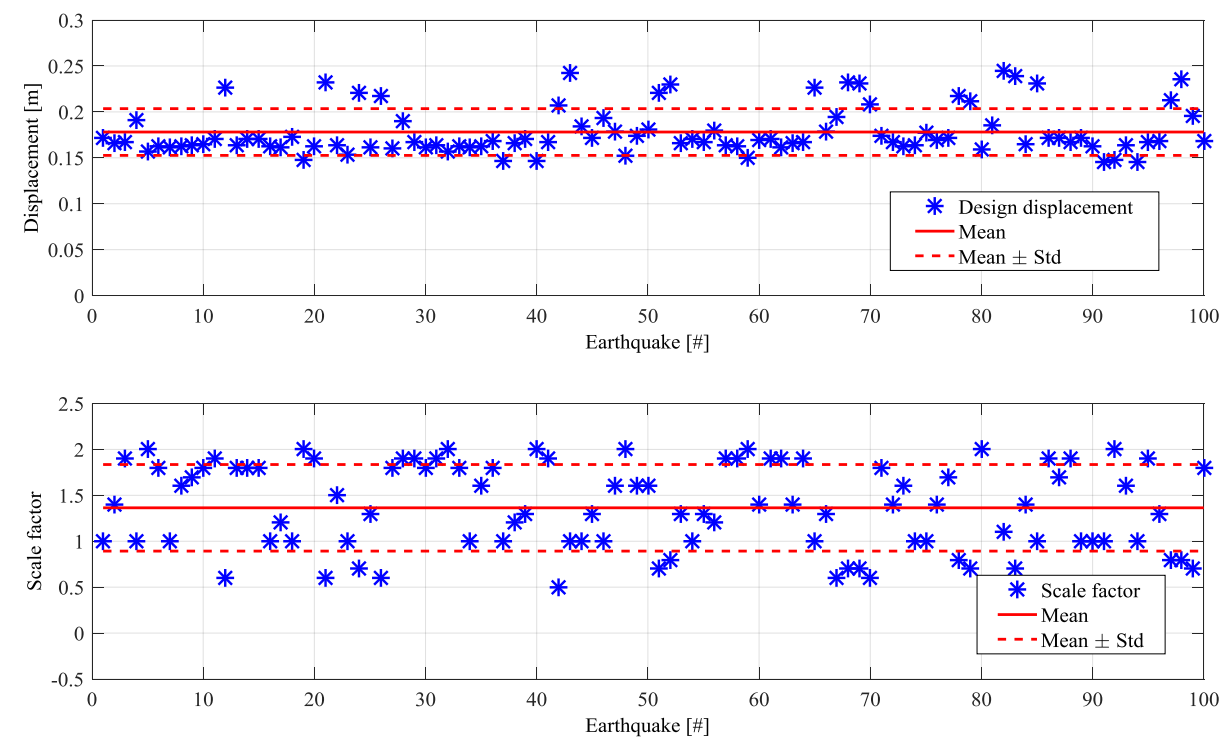

Figure 3: Selection of bidirectional events for statistical study.

In Figure 4 results in terms of variation of the optimum orientation angle are shown for acceleration (top) and displacement (bottom) approaches, by considering full-range (left) and 
Italian Building Code I.B.C. (right), as a function of increasing damping ratio, with respect to the reference value at $5 \%$ damping.
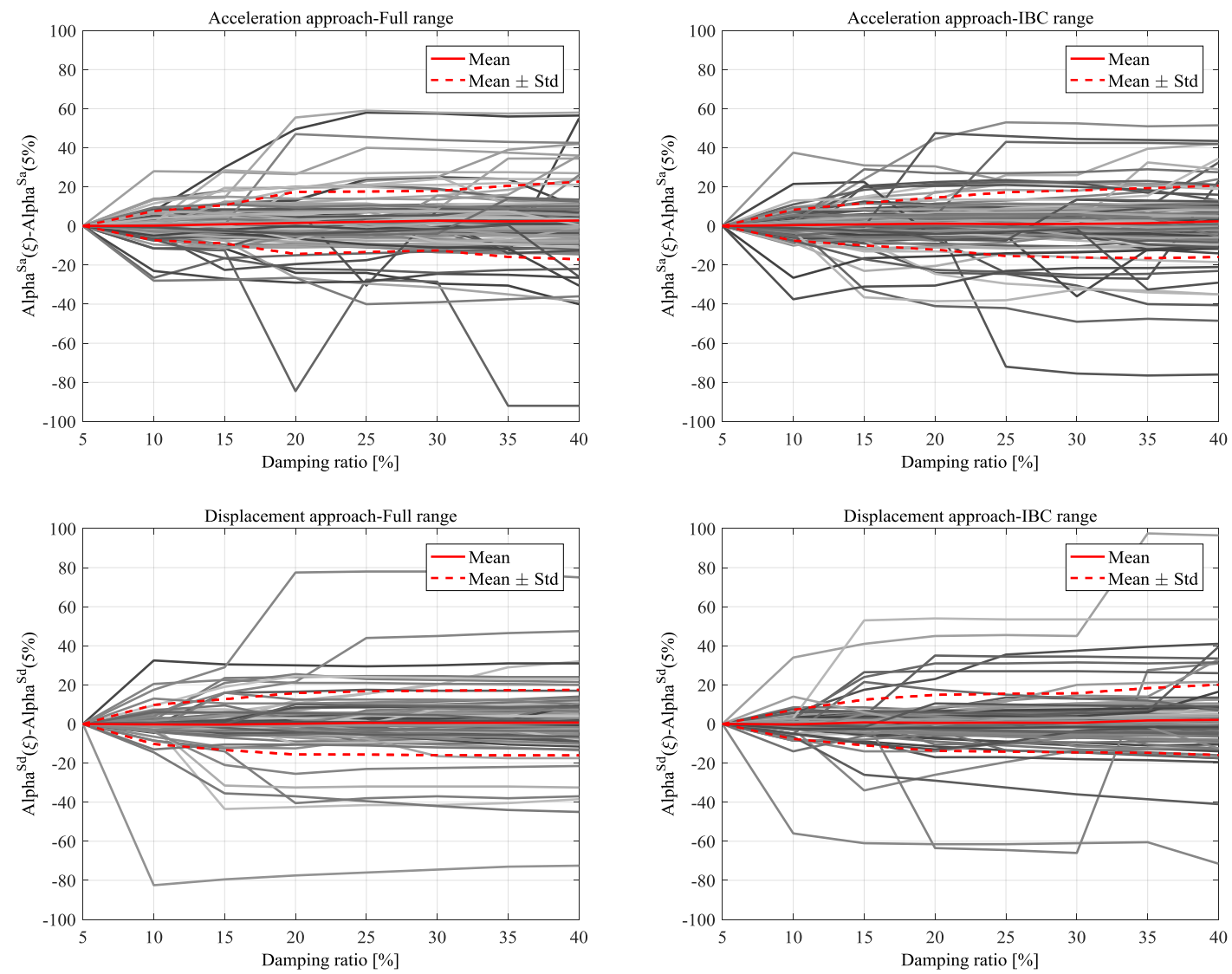

Figure 4: Variation of the orientation angle vs damping ratio: full range (left) and I.B.C. (right) results.

Statistical results show that for damping ratios higher than 5\% the optimum orientation angle is averagely constant, with an increasing variability, up to an asymptotic range of variation $\left( \pm 20^{\circ}\right)$ : this aspect allows to compute the equivalent radial seismic input, by assuming $5 \%$ damping, as for spectrum-compatibility check of a records set, without any more refined evaluation of the damping ratio, which is not a priori known for highly non-linear behaviors. Approximately same variability is noticed for both acceleration and displacement approaches. Nonetheless, in some case a higher damping ratio leads to non-negligible changes in the optimum orientation angle.

In Figure 5 error variation values of the optimum orientation angle are shown for acceleration (top) and displacement (bottom) approaches, by considering full-range (left) and Italian Building Code I.B.C. (right), as a function of increasing damping ratio, with respect to the reference value at 5\% damping. Results returned by I.B.C. assumptions have been multiplied by $\gamma$, which represents the ratio between the average values at $5 \%$ damping for I.B.C. and full range respectively, aiming at comparing the accuracy level of the considered period ranges. 

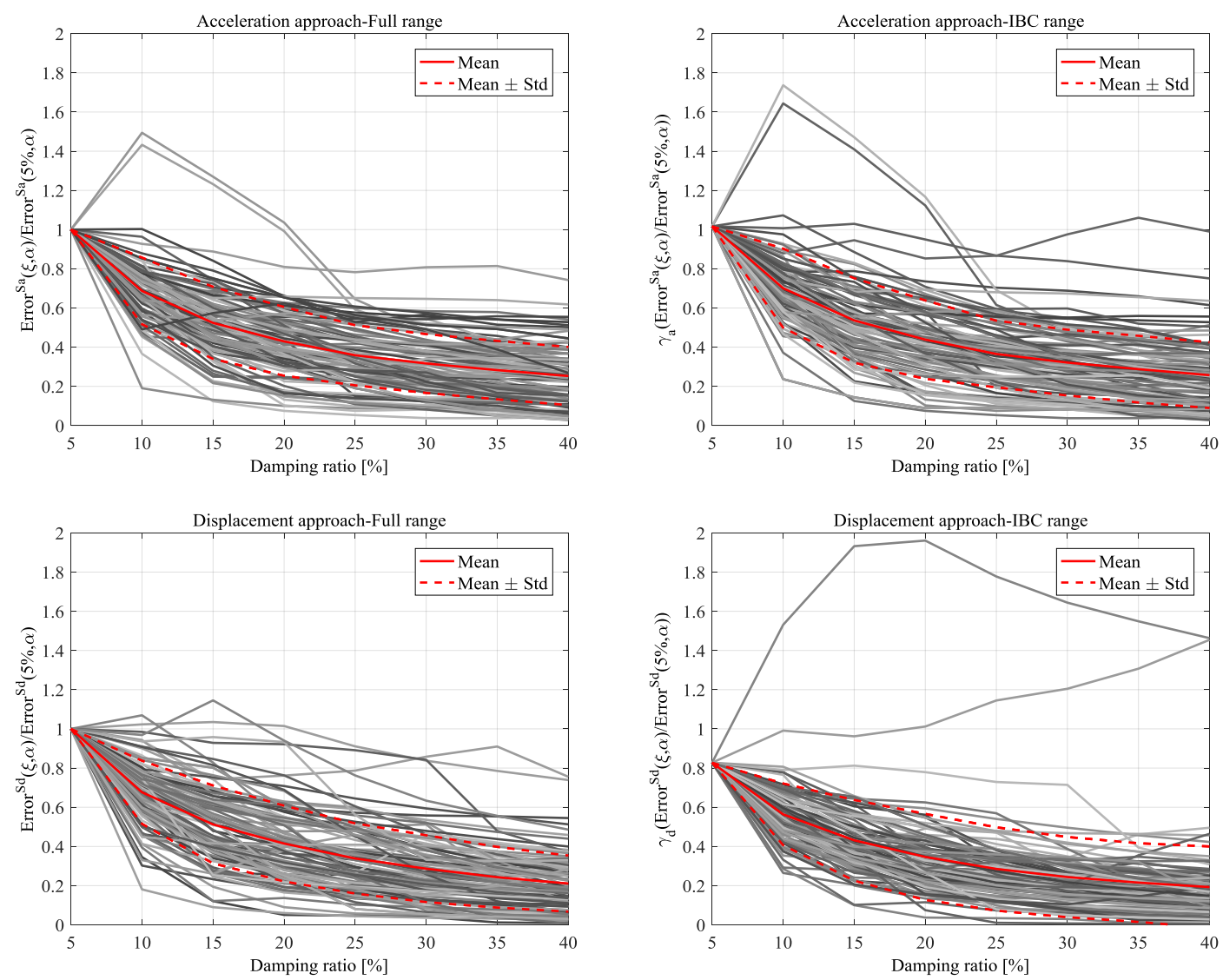

Figure 5: Error variation vs damping ratio: full range (left) and I.B.C. (right) results.

As expected, the Mean Square Error (MSE) between the reference bidirectional and the equivalent radial response spectra decreases as the damping ratio increases: for both the considered period ranges, the MSE at $5 \%$ of damping is reduced of $50 \%$ if a damping ratio of approximately $15 \%$ is assumed: thus, if base-isolated structures are analyzed, a damping ratio higher than 5\% would surely return more accurate results. Furthermore, it can be assessed that a smaller period range leads to lower MSE values, if the displacement approach is applied.

Finally, mean curves of full-range results are compared to the ones related to the I.B.C. period range, in order to investigate if a smaller period range produces the same variability of the full range case (Figure 6).

Results show that the considered period ranges lead to approximately the same variability of the optimum orientation angle for both the approaches: averagely, the orientation angle does not change for higher values of damping ratio, in comparison to $5 \%$ case, with maximum variation range bounded between $\pm 20^{\circ}$. 

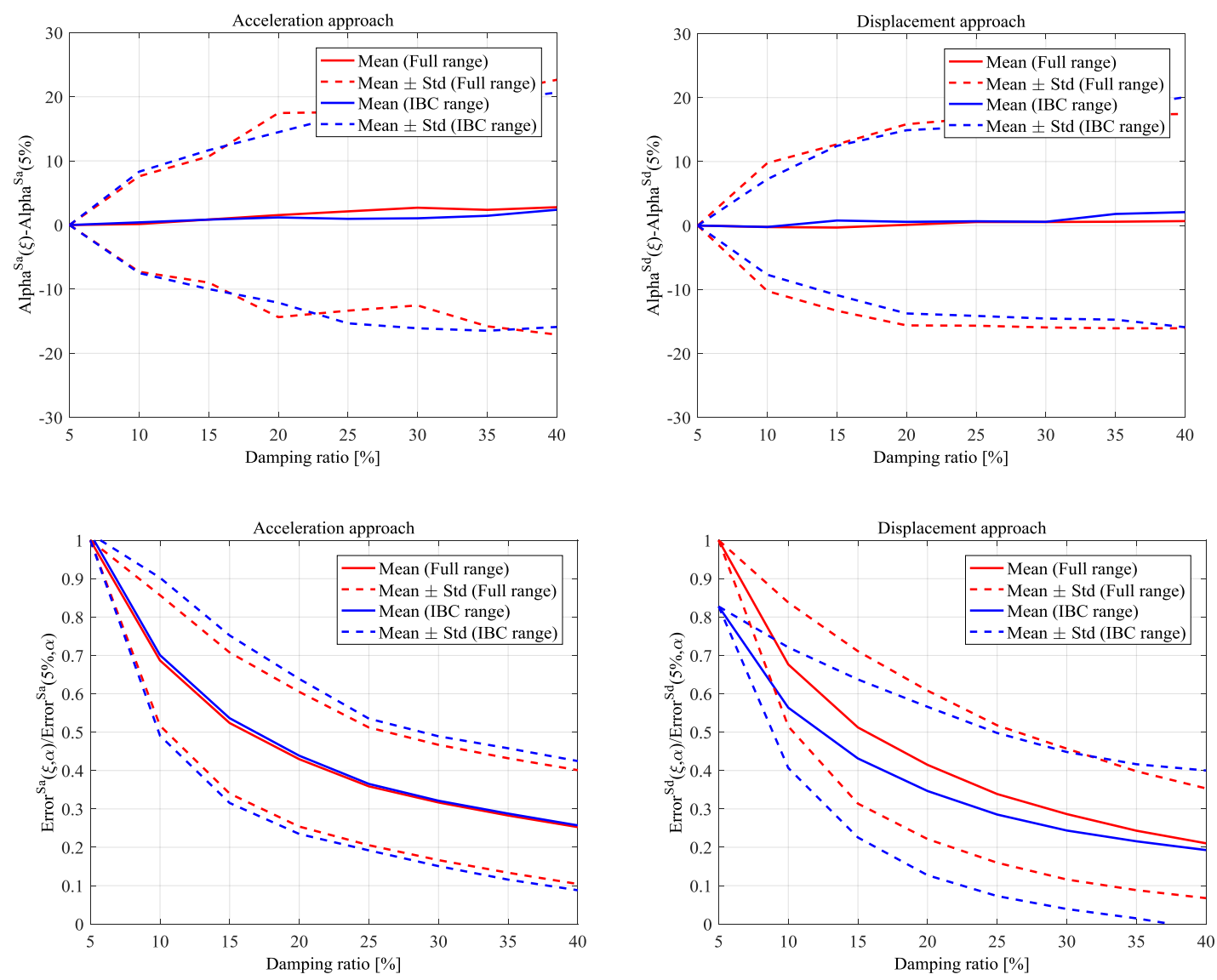

Figure 6: Period ranges comparison for orientation angle (top) and error (bottom): acceleration (left) and displacement (right) approaches.

MSE results show that the acceleration approach leads to the same mean curve, with comparable variability; on the other hand, the displacement approach with I.B.C. period range returns lower MSE values at all damping ratio values, even though a slightly larger variability can be noticed.

\section{CASE STUDY STRUCTURE}

In this work a case study structure has been analyzed, by considering bidirectional rather than equivalent radial seismic events, according to both acceleration and displacement approaches. The structural system consists of a three stories reinforced concrete frame structure, base isolated by means of Concave Surface Slider devices; the structure has been implemented in a F.E.M. software, by considering a linear-elastic model for all the structural elements, and the actual constitutive law of CSS isolators for the non-linear links, which represent the isolation devices: such a link does account for the biaxial interaction of the directions of motion, so the main frictional force for all the isolators is stepwise projected along the main directions of motion, when bidirectional earthquakes are applied. In Figure 7 the structural F.E.M. is shown. 


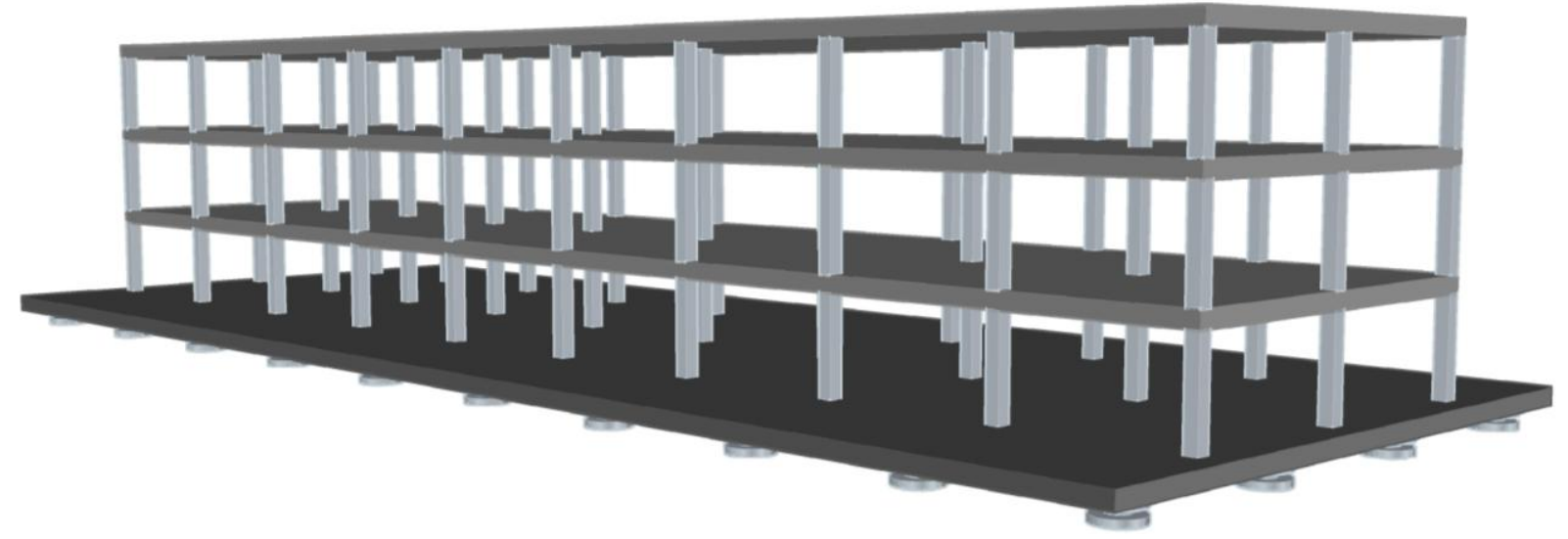

Figure 7: Case study structure

The building is located on a reinforced concrete slab, which has been implemented by using shell-thin elements, $0.5 \mathrm{~m}$ thick, in order to model both the in-plane and out-of-plane behavior.

In Table 1 main characteristics of the analyzed structural systems are listed, in terms of building mass, isolated plate mass, "fixed-base" fundamental periods and behavior factor.

\begin{tabular}{ccccc}
\hline $\mathrm{M}_{\mathrm{s}}[\mathrm{t}]$ & $\mathrm{M}_{\mathrm{p}}[\mathrm{t}]$ & $\mathrm{T}_{\mathrm{FB}, \mathrm{x}}[\mathrm{s}]$ & $\mathrm{T}_{\mathrm{FB}, \mathrm{y}}[\mathrm{s}]$ & $\mathrm{q}$ \\
\hline 1800 & 1200 & 0.42 & 0.56 & 3.45 \\
\hline
\end{tabular}

Table 1: Characteristics of the structural system.

Beneath the R.C. slab, all the isolators have been placed. For all the simulations Double Concave Surface Slider devices have been considered (Figure 8).
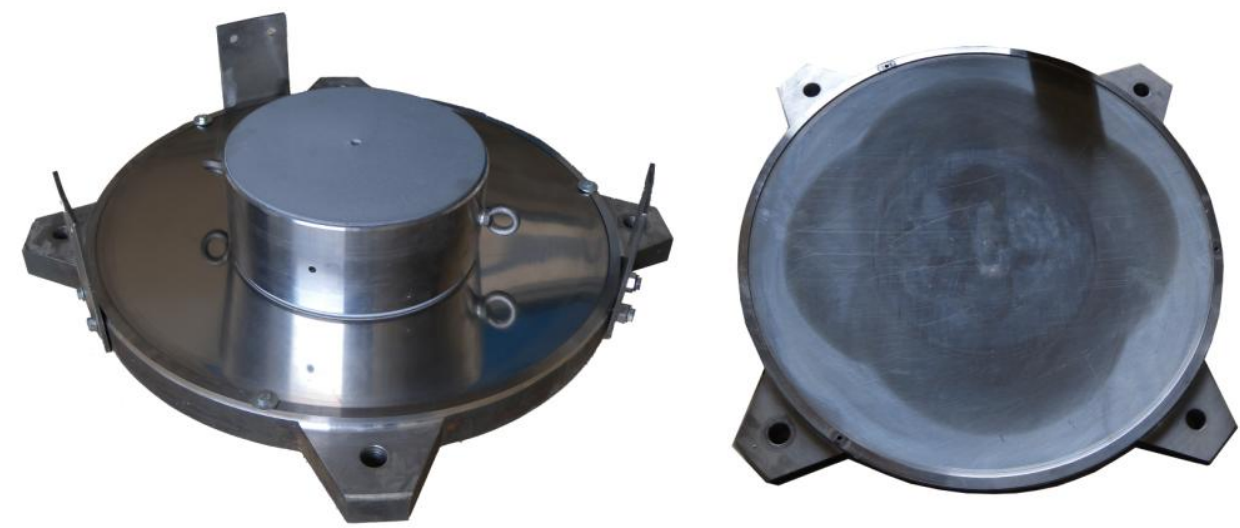

Figure 8: Double Concave Surface Slider device.

Both the sliding surfaces have the same radius of curvature $(1.6 \mathrm{~m})$, and the height of the inner non-articulated slider is equal to $120,0 \mathrm{~mm}$. Thus, the equivalent radius of curvature of a single device results $3,08 \mathrm{~m}$. For all the analyses the friction coefficient has been assumed to be constant with respect to both sliding velocity and contact pressure; furthermore, no cyclic decay has been considered, due to heating phenomena at the sliding interfaces [7, 14]. Hence, the friction coefficient is constantly equal to 7\%, according to the outcomes of a testing campaign, carried out at the EUCENTRE TREES-Lab (Pavia) [13] on a full scale device, and given dimensions of the sliding materials and vertical reactions of the isolators [7]. 
Six bidirectional events have been applied, and equivalent radial accelerograms as well, computed with both acceleration and displacement approaches. An initial value of 5\% damping has been considered, for the computation of the optimum orientation angle of the radial events: results have shown that the optimum orientation angle can be considered averagely constant, for increasing values of damping ratio; moreover, for a damping ratio higher than $15 \%$ the error between the radial and the bidirectional response spectra is reduced by $50 \%$, in comparison to the 5\% damping case. Since the damping ratio is not a priori known, when friction-based isolators are considered, a reference damping ratio of 5\% has been assumed. In Table 2 the applied seismic events have been summarized.

\begin{tabular}{cccccccc}
\hline Event \# & Location & Year & Mw & $\begin{array}{c}\text { PGA }_{X} \\
{[\mathrm{~g}]}\end{array}$ & $\begin{array}{c}\mathrm{PGA}_{\mathrm{Y}} \\
{[\mathrm{g}]}\end{array}$ & $\begin{array}{c}\alpha_{\text {acc }} \\
(\xi=5 \%)\end{array}$ & $\begin{array}{c}\alpha_{\text {disp }} \\
(\xi=5 \%)\end{array}$ \\
\hline 1 & L'Aquila & 2009 & 6.3 & 0.31 & 0.33 & 40 & 40 \\
2 & Friuli & 1976 & 5.9 & 0.30 & 0.48 & 112 & 117.5 \\
3 & Irpinia & 1980 & 6.9 & 0.21 & 0.32 & 61.5 & 74.5 \\
4 & Emilia & 2012 & 6 & 0.23 & 0.29 & 95 & 74.5 \\
5 & Loma Prieta & 1989 & 6.9 & 0.32 & 0.28 & 42 & 32.5 \\
6 & Tirana & 1988 & 5,9 & 0.22 & 0.36 & 65 & 62.5 \\
\hline
\end{tabular}

Table 2: Characteristics of applied seismic events.

\section{ANALYSES RESULTS}

In what follows results are shown, in terms of trajectories of the isolation system, and vectorial displacement, absolute acceleration and shear profiles of the whole structural system, by considering bidirectional rather than equivalent radial events, with both acceleration (Sa) and displacement (Sd) approaches. In Figure 9 trajectories of the isolation system are reported.

It can be noted that radial motions develop along the largest size of the bidirectional trajectories in all the considered seismic events. The maximum displacement at the isolation level looks to be fairly estimated by radial signals, for both acceleration and displacement approaches, even though orientation angles at 5\% damping have been assumed: this is mainly due to the constant trend of the average orientation angle of the equivalent radial event, with respect to the damping ratio, as underlined in the statistical study (Figure 4); moreover, the error decreases as higher damping ratios are considered, so that the displacement demand of the isolation system is well approximated. In addition, radial trajectories are not perfectly linear: as can be noticed, small displacements occur orthogonally with respect to the main direction of motion, for both acceleration and displacement approaches. Precisely, the non-linear time history analyses have been performed by applying the equivalent radial accelerogram according to the optimum variation angle at 5\% of damping ratio: thus, transverse displacements are induced by the actual bi-axial interaction of the directions of motion, modeled by the non-linear links which represent all the isolators. 

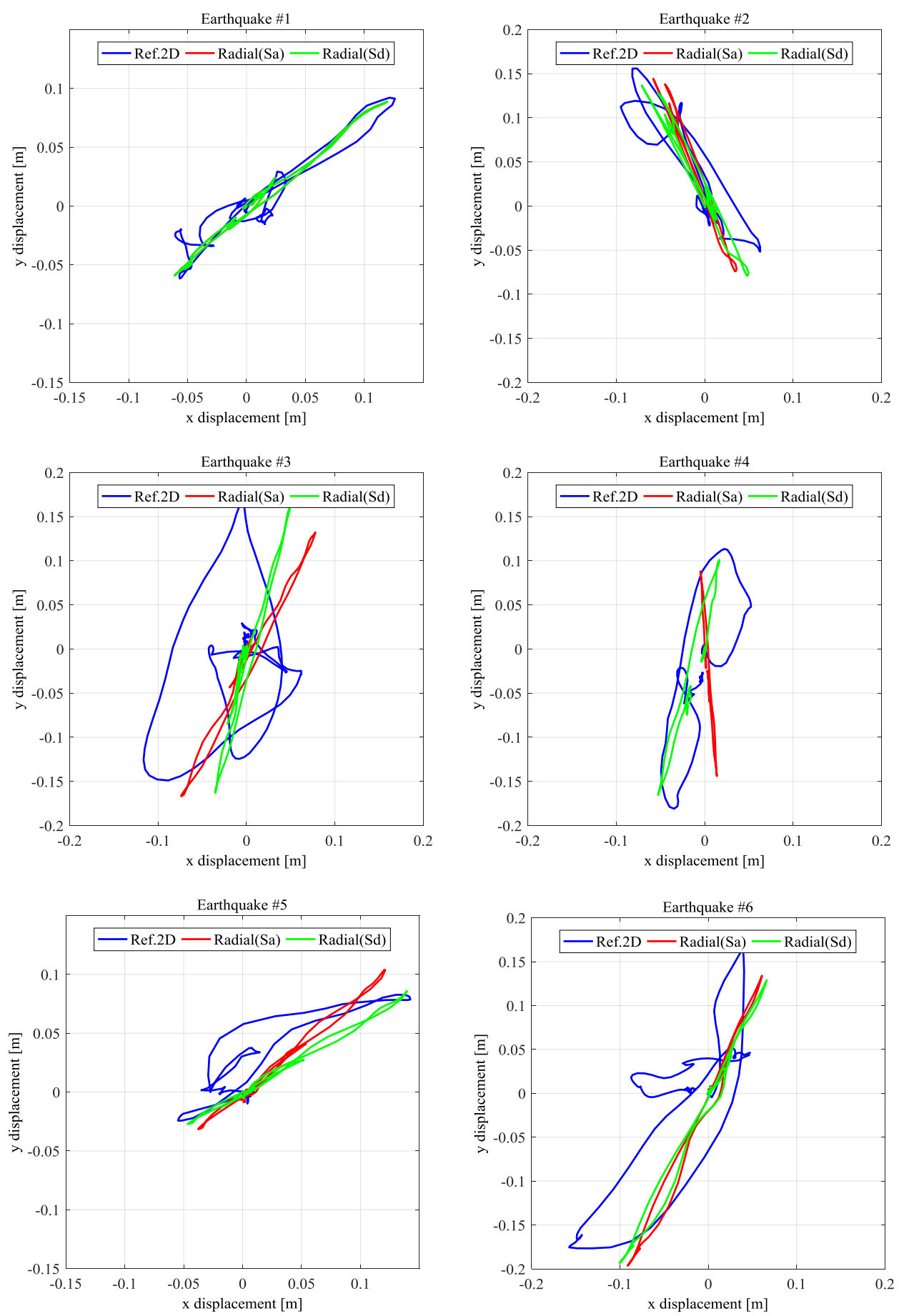

Figure 9: Analyses results: Trajectories of the isolation system.

Nonetheless, the structural system can be considered as subjected to radial motion for all the seismic events, when equivalent accelerograms are applied. 
In Figure 10 results are shown in terms of vectorial displacement profiles for all the considered seismic events.
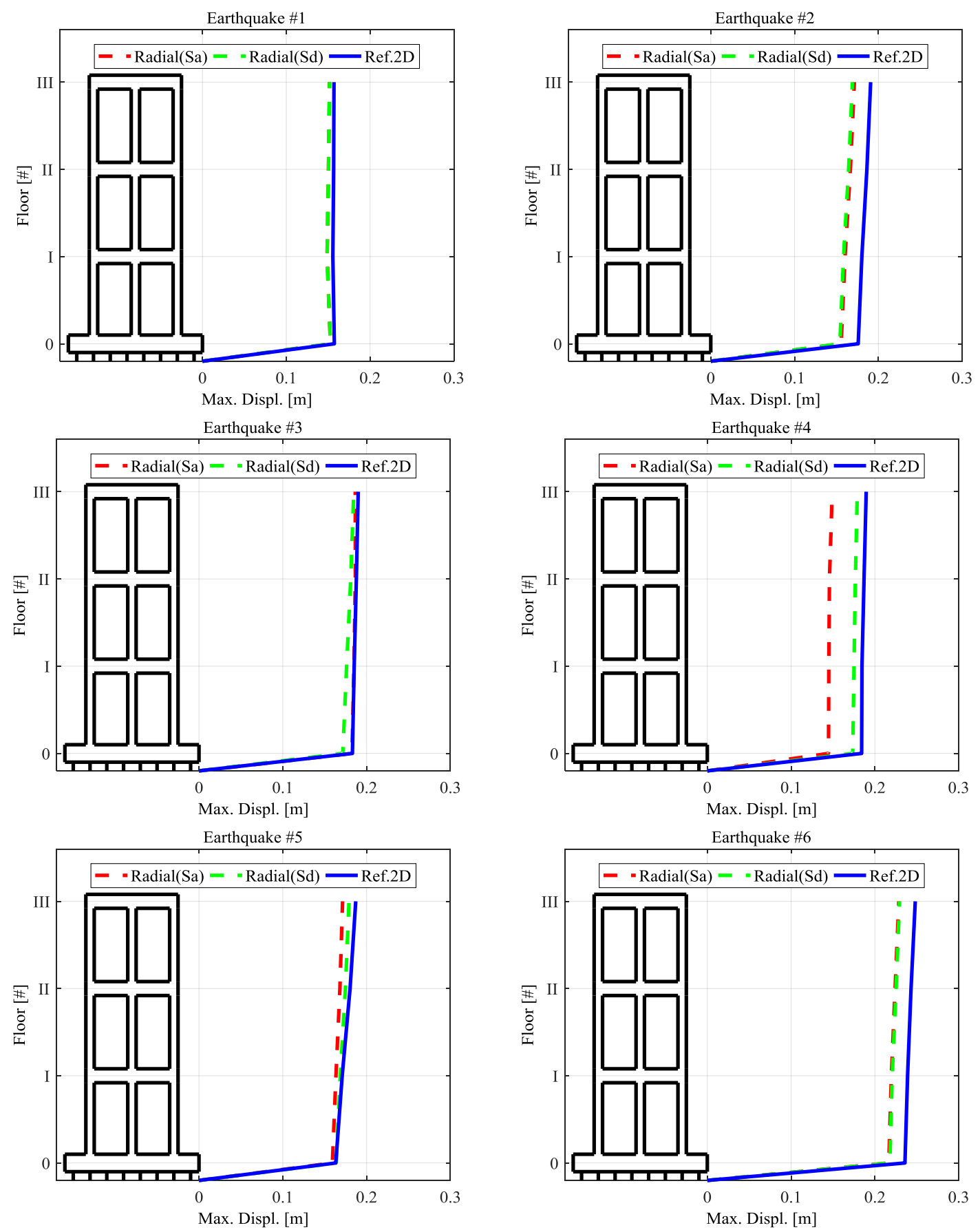

Figure 10: Analyses results: displacement profiles.

For all the considered seismic events, equivalent radial accelerograms lead to a good estimate of the displacement demand at all levels, for both acceleration and displacement approaches. In some cases, both the considered unidirectional motions return overlapped displacement profiles, due to the similar values of optimum orientation angles. Generally, a reference bidirectional event seems to lead to the highest displacement demand at all levels, whereas lower displacement values are returned by equivalent radial ground signals. 
In Figure 11 vectorial absolute acceleration values are shown at all levels of the structural system.
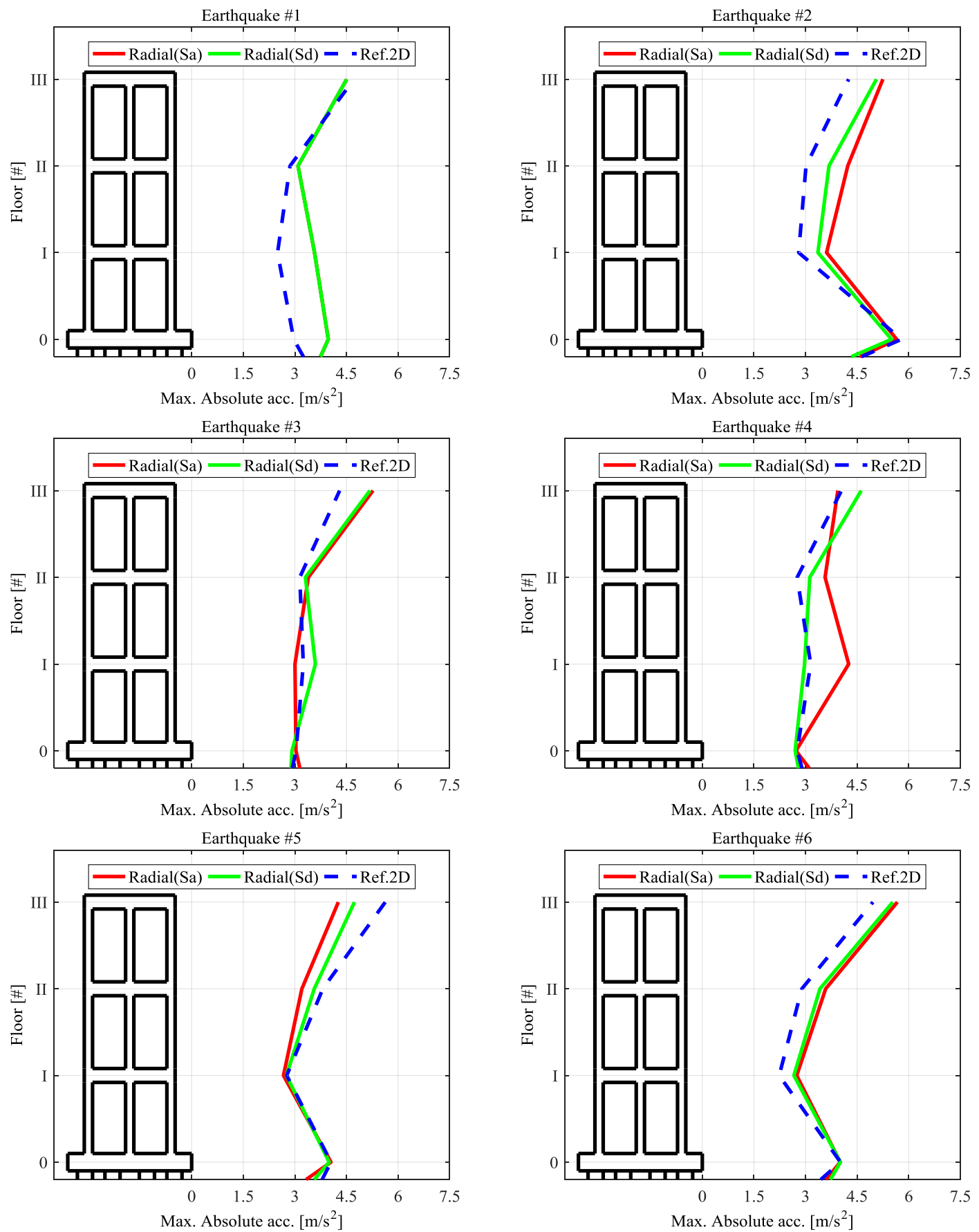

Figure 11: Analyses results: Absolute Acceleration profiles.

As for the displacement response, the acceleration response under equivalent unidirectional seismic motion well capture the reference bidirectional one for all the considered events. On the other hand, equivalent radial motions can lead to both higher and lower acceleration values, especially for the building floors levels. Furthermore, acceleration profiles in most of the cases can be considered approximately constant along the height of the structural system. 
Finally, results have been analyzed, in terms of vectorial interstorey shear values for all the structural levels (Figure 12): values have been normalized with respect to the total weight of the structural system.
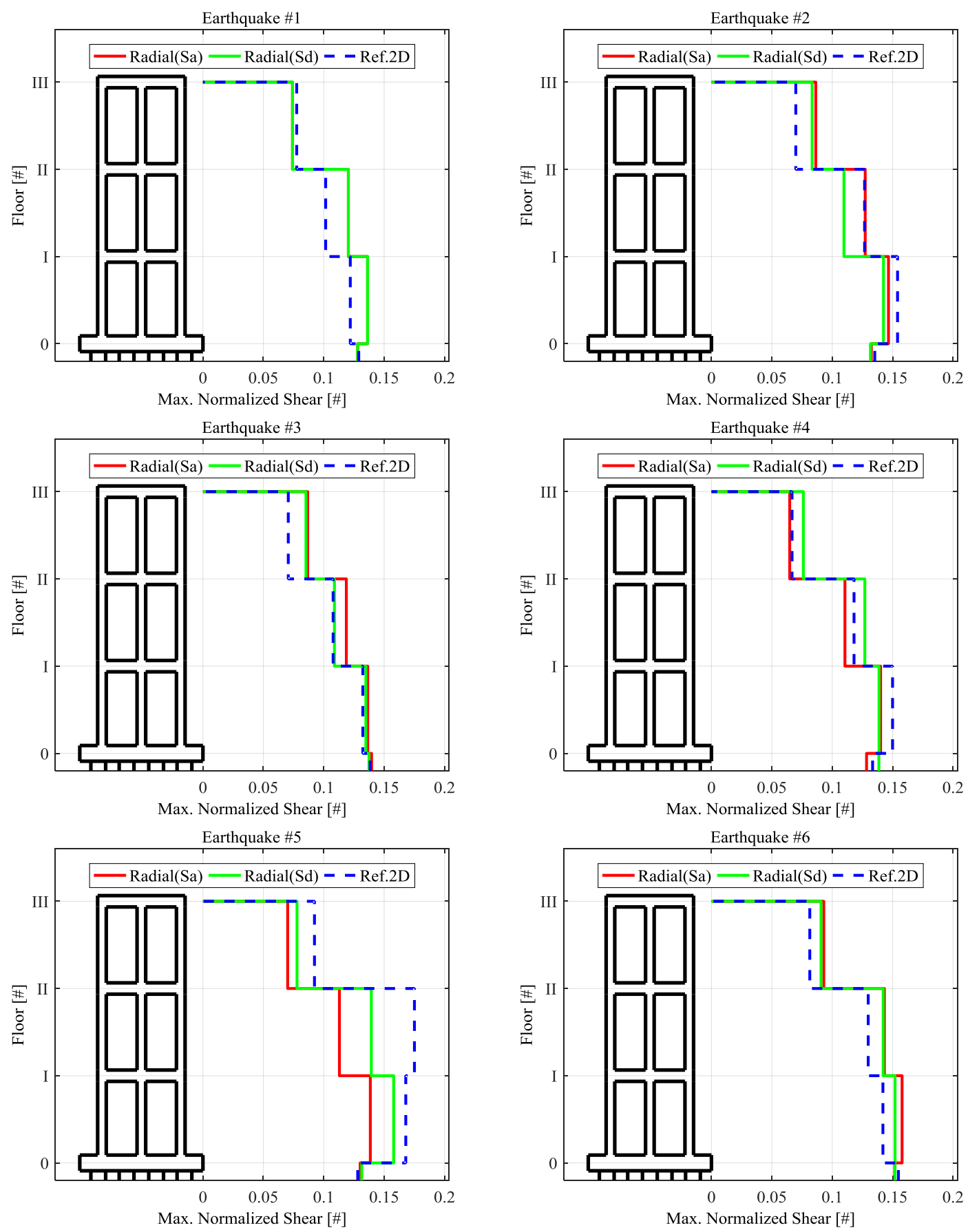

Figure 12: Analyses results: Shear profiles.

Also interstorey shear responses are properly estimated by equivalent radial events, in comparison to the bidirectional reference case. However, in some cases non-negligible variations have been found. The maximum force at the isolation level is well approximated by equivalent radial accelerograms, and the best simulation is generally returned by the displacement approach, as well as for both displacement and absolute acceleration responses. 
Thus, for base-isolated structures the displacement approach seems to be the best procedure for the computation of the equivalent unidirectional signal.

\section{CONCLUSIONS}

In the present work a more refined procedure for the computation of an equivalent radial accelerogram for bidirectional seismic events is presented. Precisely, given the reference response spectrum of a biaxial earthquake, which is represented by two individual ground acceleration time series, an optimum orientation angle is detected, by minimizing the Mean Square Error between the reference 2D and the equivalent 1D response spectra. Two different period ranges have been considered, for the computation of the MSE value, for a given damping ratio, according to the full-range, rather than the period range for spectrum compatibility ruled by the Italian Building Code (I.B.C.). Such a procedure has been applied to 100 bidirectional seismic events, in order to highlight the effect of the damping ratio on both the optimum orientation angle and the MSE. Since the presented procedure has been defined for baseisolated structure, generally represented by a variable damping ratio, as a function of the displacement demand, results have been reported in comparison to the 5\% damping ratio, which can be considered as a reference case. The optimum orientation angle is averagely independent with respect to the damping ratio, even though a non-negligible variability is found, asymptotically bounded between $\pm 20^{\circ}$. On the other hand, the Mean Square Error (MSE) decreases, if high damping ratio values are assumed, and the error at 5\% damping is reduced of $50 \%$, if damping values higher than $15 \%$ are considered. Furthermore, a smaller period range for the computation of the MSE leads to comparable variability to the full-range case, for what concerns the optimum orientation angle; however, smaller MSE values are can be found, with the I.B.C. period range.

Finally, a case study structure has been modeled by means of a F.E.M. commercial software, and bidirectional and equivalent radial seismic inputs have been applied. Six different earthquakes have been considered, by applying both the $\mathrm{x}$ and y components simultaneously, and computing an equivalent radial accelerogram with acceleration and displacement approaches, by assuming 5\% damping ratio. Results have been analyzed in terms of trajectory of the isolation system, vectorial displacement, absolute acceleration and interstorey shear profiles. The displacement demand of the bidirectional trajectory of the isolation system seems to be well captured by the equivalent radial events, for both acceleration and displacement approaches, and small variabilities are found. Concerning displacement profiles, the reference bidirectional case returns the highest displacement demand at all levels and for all the considered seismic events, and better results are achieved if the displacement approach is applied. Regarding both absolute acceleration and interstorey shear responses, the equivalent radial accelerograms lead to good estimates of the bidirectional profiles, at all levels; on the other hand, the bidirectional response is no longer the upper bound of the equivalent unidirectional ones, as for the displacement profiles. Also the maximum force at the isolation level is well approximated by the equivalent single component events: thus, the proposed procedure allows to reproduce approximately the same effects of a general bidirectional seismic event, by applying a single component ground motion, which is computed by assuming 5\% damping ratio for the optimum orientation angle; if base-isolated structures are modeled, higher damping ratio values will be found during Non Linear Time History Analysis (NLTHA), so that smaller MSE values will be averagely experienced, with no significant changes in the optimum orientation angle. Further research is needed on the topic, especially for what concerns isolation devices with smaller damping levels, rather than common fixed-base structures. 


\section{REFERENCES}

[1] Beyer K, Bommer J (2007), Selection and Scaling of Real Accelerograms for BiDirectional Loading: A review of current practice and code provisions. Journal of Earthquake Engineering, 11, 13-45, DOI: 10.1080/13632460701280013.

[2] Casarotti C, Furinghetti M, Pavese A, Evaluation of response of an isolated system based on Double Curved Surface Slider. Computational method in Earthquake Engineering, 30, pp 397-416, 2013, DOI: 10.1007/978-94-007-6573-3_19.

[3] CEN, Comité Européen de Normalisation TC 340, European Code UNI EN 15129:2009 Anti-seismic devices. Brussels, 2009.

[4] Fenz D, Constantinou MC, Behaviour of the double concave friction pendulum bearing. Earthquake Engineering And Structural Dynamics, 35,1403-1424, 2006.

[5] Furinghetti M, Casarotti C, Pavese A, Investigation of the consequences of mounting laying defects for curved surface slider devices under general seismic input. Journal of Earthquake Engineering, Under review.

[6] Furinghetti M, Pavese A (2017) Equivalent Uniaxial Accelerogram for Css-Based Isolation Systems Assessment under Two-Components Seismic Events. Mechanics Based Design of Structures and Machines, DOI: 10.1080/15397734.2017.1281145.

[7] Furinghetti M, Pavese A (2015), Numerical Assessment on the Seismic Response of a Base-Isolated Building under Bi-Directional Motion. 5th ECCOMAS Thematic Conference on Computational Methods in Structural Dynamics and Earthquake Engineering, Crete Island, Greece, May 25-27, 2015.

[8] Grant D, Diaferia R (2012), Assessing adequacy of spectrum-matched ground motions for response history analysis. Earthquake Engineering and Structural Dynamics, 42, 1265-1280, DOI: 10.1002/eqe.2270.

[9] Iervolino I, Galasso C, Cosenza E (2009), REXEL: computer aided record selsction for code-based seismic structural analysis. Bulletin of Earthquake Engineering, DOI: 10.1007/s10518-009-9146-1.

[10] Khoshnoudian F, Hagdoust VR, Response of pure-friction sliding structures to three components of earthquake excitation considering variations in the coefficient of friction. Scientia Iranica, 16, 429-442, 2009.

[11] Lomiento G, Bonessio N, Benzoni G, Concave sliding isolator's performance under multi-directional excitation. Ingegneria Sismica, 30:3,17-32, 2013.

[12] Mosqueda G, Whittaker AS, Fenves GL, Characterization and modeling of friction pendulum bearings subjected to multiple components of excitation. Journal of Structural Engineering, 130, 433-442, 2004.

[13] Peloso S, Pavese A, Casarotti C (2012), EUCENTRE TREES lab: Laboratory for training and research in earthquake engineering and seismology, Geotechnical, Geological and Earthquake Engineering, 20,65-81, DOI: 10.1007/978-94-007-1977-4_4.

[14] Quaglini V, Bocciarelli M, Gandelli E, Dubini P (2014), Numerical Assessment of Frictional Heating in Sliding Bearings for Seismic Isolation. Journal of Earthquake Engineering, 18(8), 1198-1216, DOI: 10.1080/13632469.2014.924890. 